\title{
Molecular and phylogenetic characterization of bovine coronavirus virus isolated from dairy cattle in Central Region, Thailand
}

\author{
Kanokwan Singasa $^{1}$ - Taweesak Songserm ${ }^{2} \cdot$ Preeda Lertwatcharasarakul $^{2}$. \\ Pipat Arunvipas ${ }^{1}$ (B)
}

Received: 4 February 2017 / Accepted: 5 July 2017 /Published online: 18 July 2017

(C) Springer Science+Business Media B.V. 2017

\begin{abstract}
Bovine coronavirus (BCoV) is involved mainly in enteric infections in cattle. This study reports the first molecular detection of $\mathrm{BCoV}$ in a diarrhea outbreak in dairy cows in the Central Region, Thailand. BCoV was molecularly detected from bloody diarrheic cattle feces by using nested PCR. Agarose gel electrophoresis of three diarrheic fecal samples yielded from the 25 samples desired amplicons that were 488 base pairs and sequencing substantiated that have $\mathrm{BCoV}$. The sequence alignment indicated that nucleotide and amino acid sequences, the three TWD isolated in Thailand, were more quite homologous to each other (amino acid at position 39 of TWD1, TWD3 was proline, but TWD2 was serine) and closely related to OK-0514-3strain (virulent respiratory strain; RBCoV).The amino acid sequencing identities among TWD1, TWD2,TWD3, and OK-0514-3 strain were 96.0 to $96.6 \%$, those at which T3I, H65N, D87G, H127Y, andQ136R were changed. In addition, the phylogenetic tree of the hypervariable region S1subunit spike glycoprotein $\mathrm{BCoV}$ gene was composed of three major clades by using the 54 sequences generated and showed that the evolutionally distance, TWD1, TWD2, and TWD3 were the isolated group together and most similar to OK-0514-3 strain (98.2 to 98.5\% similarity). Further study will develop ELISA assay for serologic detection of winter dysentery disease.
\end{abstract}

Pipat Arunvipas

fvetpia@ku.ac.th

1 Department of Large Animal and Wildlife Clinical Science, Faculty of Veterinary Medicine, Kasetsart University, Kampangsaen Campus, 1 Malaiman Road, Kamphaeng Saen, Kamphaeng Saen District, Nakhon Pathom 73140, Thailand

2 Department of Pathology, Faculty of Veterinary Medicine, Kasetsart University, Kampangsaen Campus, Nakhon Pathom, Thailand
Keywords Bovine coronavirus · Dairy cattle $\cdot$ Nested PCR . Winter dysentery disease

\section{Introduction}

Bovine coronavirus $(\mathrm{BCoV})$ is a single-stranded, non-segmented, positive sense RNA genome of 27 to $32 \mathrm{~kb}$. BCV virion is enveloped and pleomorphic to spherical in shape about $80-200 \mathrm{~nm}$ in diameter. It is classified in the order Nidovirales, family Coronaviridae, subfamily Coronavirinae, genus Betacoronavirus (Group 2 Coronavirus) subgroup 2A (Graham et al. 2013). Its genome includes 13 open reading frames (ORFs) flanked by $5^{\prime}$ and $3^{\prime}$ untranslated regions. The genome of $\mathrm{BCoV}$ contains of five structural proteins and non-structural proteins. Five major structural proteins are encoded within the genomic RNA such as hemagglutinin-esterase (HE) protein (ORF3), spike (S) glycoprotein (ORF4), small membrane (E) protein (ORF8), transmembrane (M) protein (ORF9), and nucleocapsid (N) protein (ORF10) (Chouljenko et al. 2001; Masters 2006).

The S protein is a $180-\mathrm{kDa}$ glycoprotein, length $4038 \mathrm{bp}$ (nn 2152-6243), type 1 viral fusion protein on the viral surface, playing an important role in induction of neutralizing antibodies and cleaved at the amino acid position 768-769 in two subunits: S1 subunit and S2 subunit (Yoo and Deregt 2001). The S protein forms club-shaped structure which has amino-terminal S1 receptor binding unit that is the bulbous part, whereas the carboxyl-terminal S2 membrane fusion unit is the stalk part (Bosch et al. 2003). Moreover, S1 subunit has the downstream hypervariable region (HVR) that is different in each strain, but S2 subunit is conserved among strains (Weiss and Martin 2005). 
Table 1 Primer sequences along with expected PCR product size and the thermal cycling condition

\begin{tabular}{|c|c|c|c|c|c|}
\hline Primer name & \multicolumn{2}{|l|}{ Primer sequence } & Product size (bp) & \multicolumn{2}{|l|}{ Reference } \\
\hline S1HS & \multicolumn{2}{|c|}{ (5')-CTATACCCAATGGTAGGA-( $\left.3^{\prime}\right)$} & 885 & \multicolumn{2}{|c|}{ Brandao et al. (2003) } \\
\hline S1HA & \multicolumn{2}{|c|}{ (5')-CTGAAACACGACCGCTAT-(3') } & & & \\
\hline S1N & \multicolumn{2}{|c|}{ (5')-GTTTCTGTTAGCAGGTTTAA-(3') } & 488 & & \\
\hline S1NA & \multicolumn{2}{|c|}{ (5')-ATATTACACCTATCCCCTTG-(3') } & & & \\
\hline \multicolumn{6}{|c|}{ Thermal cycling condition } \\
\hline & Pre-denaturation & Denaturation & Primer annealing & Primer extension & Final extension \\
\hline Outer primers & $94{ }^{\circ} \mathrm{C} ; 180 \mathrm{~s}$ & $\begin{array}{l}94^{\circ} \mathrm{C} ; 60 \mathrm{~s} \\
\times 35 \text { cycles }\end{array}$ & $53.4^{\circ} \mathrm{C} ; 90 \mathrm{~s}$ & $72{ }^{\circ} \mathrm{C} ; 60 \mathrm{~s}$ & $72{ }^{\circ} \mathrm{C} ; 600 \mathrm{~s}$ \\
\hline Internal primers & $94{ }^{\circ} \mathrm{C} ; 180 \mathrm{~s}$ & $\begin{array}{l}94^{\circ} \mathrm{C} ; 60 \mathrm{~s} \\
\times 25 \text { cycles }\end{array}$ & $58.4^{\circ} \mathrm{C} ; 90 \mathrm{~s}$ & $72{ }^{\circ} \mathrm{C} ; 60 \mathrm{~s}$ & $72{ }^{\circ} \mathrm{C} ; 600 \mathrm{~s}$ \\
\hline
\end{tabular}

$\mathrm{BCoV}$ is the cause of winter dysentery disease, that is a hemorrhagic enteritis with anorexia, dehydration, emaciation, and suffering severely from decreasing production in adult dairy cattle. Moreover, $\mathrm{BCoV}$ causes watery diarrhea in newborn calves whose range ages from 1 week to 3 months (Blowey and Weaver 2011). BCoV infection is a serious cause of economic losses to the dairy industry around the world (Saif 2004). Besides, the maximum decrease in milk production ranges around $10 \%$ and may last for 1-2 weeks, after that milk production levels are regained in mild epidemics of $\mathrm{BCoV}$, but in severe epidemics, milk production decreases to $30 \%$ and continues up to 1 month (Radostits et al. 2007).

$\mathrm{BCoV}$ was first reported by Mebus in USA (Mebus et al. 1972, 1973), which is the Mebus strain, and by Kanno, T. in Asia (Kanno et al.2007), which is the Kakegawa strain; Aiumlamai et al. (1992) reported only prevalence of bovine coronavirus antibody in bulk tank milk samples at $93 \%$ in Muaklek area, Saraburi Province, Thailand, but there are no reports of bovine coronavirus molecular diagnosis in cattle. This study reveals the molecular and phylogenetic characterization of bovine coronavirus by molecular methodology in dairy cattle in Thailand.

\section{Materials and methods}

\section{Sample collection}

Twenty-five Holstein-Friesian cattle in six farms that had clinically suspected cattle in Central Region, Thailand, were collected whole bloods and feces. Some cattle showed clinical signs such as lethargy, bloody diarrhea, watery diarrhea, milk production decreasing, and death in some cows. All samples were stored at $-80{ }^{\circ} \mathrm{C}$ until processing.

\section{RNA extraction and cDNA synthesis}

RNA were extracted from whole bloods and feces of all cattle using FavorPrep ${ }^{\mathrm{TM}}$ Viral Nucleic Acid Extraction Kit I (Favorgen ${ }^{\circledR}$ ) according to the manufacturer's instructions; then $11 \mathrm{ul}$ of each RNA was added into a PCR tube containing of 4 ul of $5 \times$ reaction buffer, 2 ul of Random hexamer primer, $2 \mathrm{ul}$ of $10 \mathrm{mM}$ dNTP, $0.5 \mathrm{ul}$ of RNase Inhibitor, and $1 \mathrm{ul}$ of Thermo Scientific RevertAid ${ }^{\mathrm{TM}}$ Reverse Transcriptase; mix gently and spin down; then incubated $10 \mathrm{~min}$ at $25^{\circ} \mathrm{C}$ followed by $60 \mathrm{~min}$ at $42^{\circ} \mathrm{C}$ and terminate the reaction by heating at $70{ }^{\circ} \mathrm{C}$ for $10 \mathrm{~min}$ in a thermal cycler (Bio-Rad T100 ${ }^{\mathrm{TM}}$, Bio$\operatorname{Rad}()$.

\section{PCR primers and conditions}

Two pairs of primers were followed Brandao et al. (2003) that conserved regions flanking the hypervariable region of the $\mathrm{S}$ gene (GenBank accession no. M31053): outer primers (sense S1HS 5'-CTATACCCAATGGTAGGA-3' and anti-sense S1HA 5'-CTGAAACACGACCGCTAT-3') with a predicted 885-bp-long product (nn 1204 to 2088 from the $\mathrm{S}$ gene) and internal primers (sense S1N 5'-GTTTCTGTTAGCAG GTTTAA-3' and anti-sense S1NA 5'-ATATTACACCTATC CCCTTG-3') with a predicted 488-bp-long product (nn 1329 to 1816 from the $\mathrm{S}$ gene) (Brandao et al. 2003). The expected PCR product size alongside the thermal cycling conditions of the primers was given in Table 1 . PCR reactions were set up into 20-ul volume containing 18 ul PCR master mix (2.5 units Taq DNA polymerase (Invitrogen ${ }^{\mathrm{TM}}$ ) in $1 \times \mathrm{PCR}^{-\mathrm{MgCl}_{2}}$ buffer, $\left.1.5 \mathrm{mM} \mathrm{MgCl}_{2}, 0.2 \mathrm{mM} \mathrm{dNTP}\right), 0.5 \mathrm{uM}$ of each primer, 2 ul of the DNA template , and total volume was made up to 20 ul. DNA from the whole bloods as well as from the feces of both calves and cows was used. The expected PCR products 
Table 2 GenBank accession numbers of reference strains of subgroup 2A genus Betacoronavirus, country of origin used in neighbor-joining analysis of spike glycoprotein sequences

\begin{tabular}{|c|c|c|c|c|}
\hline Strain & Year & Country & Strain origin & $\begin{array}{l}\text { GenBank } \\
\text { accession no. }\end{array}$ \\
\hline Mebus & 1972 & USA & Enteric & U00735 \\
\hline LY-138 & 1965 & USA & Enteric & AF058942 \\
\hline ENT & 1998 & USA & Enteric & AF391541 \\
\hline LSU-94LSS-051-2 & 1994 & USA & Respiratory & AF058943 \\
\hline OK-0514-3 & 1996 & USA & Respiratory & AF058944 \\
\hline L9 & 1991 & USA & Vaccine strain & M64667 \\
\hline $182 \mathrm{NS}$ & 2000 & USA & Respiratory & DQ320764 \\
\hline $220 \mathrm{NS}$ & 1998 & USA & Respiratory & DQ320762 \\
\hline $232 \mathrm{NS}$ & 2000 & USA & Respiratory & DQ320763 \\
\hline Norden vaccine & 1991 & USA & Vaccine strain & M64668 \\
\hline F15 & 1979 & France & Enteric & D00731 \\
\hline Quebec & 1972 & Canada & Winter Dysentery & AF220295 \\
\hline BCQ7373 & 1992 & Canada & Winter Dysentery & AF239306 \\
\hline BCQ1523 & 1989 & Canada & Enteric & AF239307 \\
\hline BCQ2590 & 1992 & Canada & Winter Dysentery & AF239317 \\
\hline BCQ3994 & 2001 & Canada & Respiratory & AF339836 \\
\hline BCO44175 & 2000 & Canada & Respiratory & AF239309 \\
\hline BCO43277 & 2000 & Canada & Respiratory & AF239308 \\
\hline BCQ571 & 1989 & Canada & Enteric & АH010363 \\
\hline BCQ9 & 1989 & Canada & Enteric & U06091 \\
\hline BCQ20 & 1989 & Canada & Enteric & U06092 \\
\hline BCQ2070 & 1989 & Canada & Enteric & U06090 \\
\hline BR-UEL1 & 2004 & Brazil & Enteric & DQ479421 \\
\hline BR-UEL2 & 2004 & Brazil & Enteric & DQ479422 \\
\hline BR-UEL3 & 2004 & Brazil & Enteric & DQ479423 \\
\hline Kakegawa & 1980 & Japan & Winter Dysentery & DQ479424 \\
\hline $\mathrm{KCD} 1$ & 2004 & South Korea & Enteric & DQ389632 \\
\hline $\mathrm{KCD} 2$ & 2004 & South Korea & Enteric & DQ389633 \\
\hline KCD4 & 2004 & South Korea & Enteric & DQ389635 \\
\hline KCD5 & 2004 & South Korea & Enteric & DQ389636 \\
\hline KCD6 & 2004 & South Korea & Enteric & DQ389637 \\
\hline $\mathrm{KCD} 7$ & 2004 & South Korea & Enteric & DQ389638 \\
\hline $\mathrm{KCD} 8$ & 2004 & South Korea & Enteric & DQ389639 \\
\hline KWD1 & 2002 & South Korea & Winter dysentery & AY935637 \\
\hline KWD2 & 2002 & South Korea & Winter dysentery & AY935638 \\
\hline KWD3 & 2002 & South Korea & Winter dysentery & AY935639 \\
\hline KWD4 & 2002 & South Korea & Winter dysentery & AY935640 \\
\hline KWD5 & 2002 & South Korea & Winter dysentery & AY935641 \\
\hline KWD7 & 2002 & South Korea & Winter dysentery & AY935643 \\
\hline KWD9 & 2002 & South Korea & Winter dysentery & AY935645 \\
\hline KWD11 & 2002 & South Korea & Winter dysentery & DQ389652 \\
\hline KWD13 & 2002 & South Korea & Winter dysentery & DQ389654 \\
\hline KWD14 & 2002 & South Korea & Winter dysentery & DQ389655 \\
\hline KWD15 & 2002 & South Korea & Winter dysentery & DQ389656 \\
\hline KWD16 & 2002 & South Korea & Winter dysentery & DQ389657 \\
\hline BC94Korean vaccine & 1994 & South Korea & Vaccine strain & EU401989 \\
\hline 0501 & 2005 & South Korea & Vaccine strain & EU686689 \\
\hline 0502 & 2005 & South Korea & Vaccine strain & EU401986 \\
\hline A3 & 1994 & South Korea & Vaccine strain & EU401987 \\
\hline SUN5 & 1994 & South Korea & Vaccine strain & EU401988 \\
\hline $\mathrm{HCoV}-\mathrm{OC} 43$ & 1967 & UK & Respiratory & $\mathrm{NC} 005147$ \\
\hline
\end{tabular}




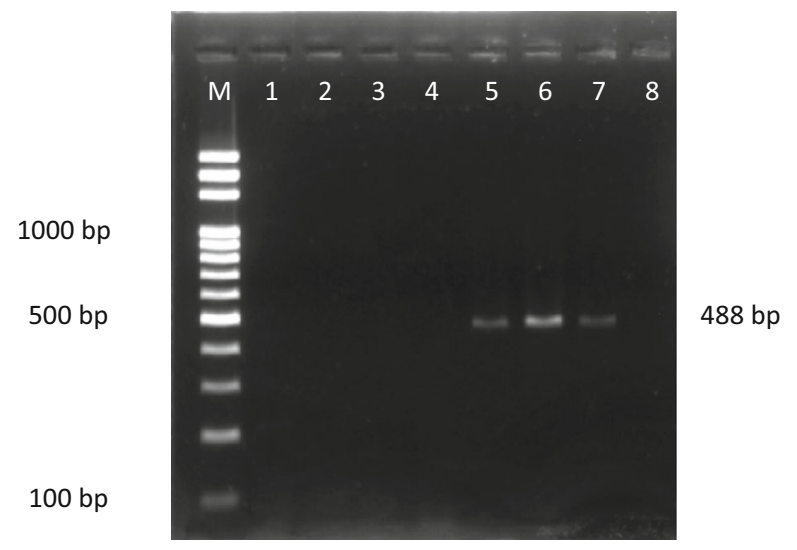

Fig. 1 Nested PCR amplification lane M: 100 bp DNA ladder(Solis BioDyne); lanes 1-3: three known positive samples using outer primers; lane 4: negative control; lanes 5-7: known positive samples using inner primers; lane 8 : negative control

were analyzed by $1.5 \%$ agarose gel electrophoresis in $1 \times$ Trisacetate-EDTA (TAE) buffer $\mathrm{pH} 8.3(40 \mathrm{mM}$ Tris, $20 \mathrm{mM}$ acetic acid, and $1 \mathrm{mM}$ EDTA), visualized with GelRed ${ }^{\mathrm{TM}}$ nucleic acid staining (Biotium) and analyzed by Gel Doc ${ }^{\mathrm{TM}}$ EZ System (Bio-Rad®).

\section{Sequencing analysis}

The PCR products from the nested PCR were purified by Thermo Scientific gene JET gel extraction kit; DNA fragments were sent to First Base Co., Ltd. (Selangor, Malaysia) for sequencing. Sequence quality analysis as performed by using BioEdit sequence alignment editor and the consensus sequences were assembled using the CAP contig assembly program of BioEdit. Finally, the sequences were submitted to the nucleotide basic local alignment search tool (BLASTn) software (http://blast.ncbi. nlm.nih.gov/) to find the most similar sequences and possible non- $\mathrm{BCoV}$ related similarities.

Table 3 Corrected $\mathrm{OD}$ values $\left(\mathrm{OD}_{\text {corr }}\right)$ and percent positivity values (PP) of TWD1-3 from SVANOVIR $®$ BCV-Ab test

\begin{tabular}{llrl}
\hline Sample name & OD $_{\text {corr }}^{\mathrm{a}}$ & $\mathrm{PP}^{\mathrm{b}}$ & Interpretation \\
\hline TWD1 & 1.277 & 84.570 & Positive \\
TWD2 & 1.820 & 128.124 & Positive \\
TWD3 & 1.771 & 124.674 & Positive \\
\hline
\end{tabular}

\footnotetext{
${ }^{a}$ The optical density (OD) values in well coated with $\mathrm{BCV}$ viral antigen are corrected by subtracting the OD values of the corresponding wells containing the control antigen. $\mathrm{OD}_{\mathrm{Corr}}=\mathrm{OD}_{\mathrm{BCV}}-\mathrm{OD}_{\text {Control }}$

${ }^{\mathrm{b}}$ All corrected OD values for the test samples as well as the negative control are related to the corrected OD value of the positive control as follows: $\mathrm{PP}=\left[\mathrm{OD}_{\mathrm{Corr}(\text { Sample/NegativeControl) }} / \mathrm{OD}_{\mathrm{Corr}}(\right.$ Positive Control $\left.)\right] \times 100$ and interpretation of serum samples; PP interpretation $<10=$ negative and $\geq 10=$ positive
}

\section{Phylogenetic analysis}

The three positive sample sequences, $50 \mathrm{BCoV}$, and 1 human coronavirus OC43 (HCoV-OC43) sequences obtained from the National Center for Biotechnology Information, USA (GenBank) (http://www.ncbi.nlm.nih.gov/genbank/), were aligned and constructed a phylogenetic tree by using the Molecular Evolutionary Genetics Analysis (MEGA, version 7). The GenBank accession numbers of $50 \mathrm{BCoV}$ and $\mathrm{HCoV}$ OC43 strains are indicated in Table 2. HCoV-OC43 strain was used as an outgroup. The phylogenetic tree was created by using the neighbor-joining method with bootstrap test (1000 replicates) based on the nucleotide sequence of the hypervariable region in $\mathrm{BCoV}$. The evolutionary distances were computed by using the Tamura 3-parameter method.

\section{GenBank accession number}

GenBank accession number KX373886, KX373887, and KX373888 were assigned to TWD1, TWD2, and TWD3, respectively. These are the sequenced 448-bp fragment.

\section{Results}

Clinical inspection of affected dairy cattle showed watery diarrhea, bloody diarrhea, dehydration, melena or occult blood in feces, decrease milk production, and death in some cows.

Agarose gel electrophoresis of three diarrheic fecal samples yielded that out of the 25 samples the desired amplicons that were 488 base pairs (Fig. 1). In addition, after having been purified, the three samples yielded agarose gel, and they were sent sequencing analysis, by using the BLASTn. The result showed that they possessed 97-99\% nucleotide identities to spike glycoprotein of bovine coronavirus.

The three cows were positive for fecal testing and also positive for the blood testing (Table 3). All blood samples analyzed the presence of the IgG antibodies to $\mathrm{BCoV}$ by commercially available indirect ELISA (SVANOVA, Biotech).

The sequence alignment by ClustalW multiple alignment of the BioEdit program indicated that nucleotide and amino acid sequences of the three TWD isolated in Thailand were high similar to each other (amino acid at position 39 of TWD1, TWD3 was proline, but TWD2 was serine) and were closely related to OK-0514-3 strain (virulent respiratory strain; RBCoV).The amino acid sequence identities among TWD1, TWD2, TWD3, and OK-0514-3 strain were 96.0 to $96.6 \%$, which of those at T3I, H65N, D87G, H127Y, and Q136R were changed (data not shown).

The phylogenetic tree of partial spike glycoprotein genes of bovine coronavirus was composed of three major clades by using the 54 sequences generated in this study from field samples, GenBank reference strains, and vaccine strains. 
Fig. 2 The phylogenetic tree of the partial $\mathrm{S}$ gene sequences of $\mathrm{BCoV}$. This tree was generated using the neighbor-joining method. Bootstrap values were obtained from 1000 replications. The scale bar represents the number of substitutions per site. TWD1-TWD3 were received from the three positive samples in Thailand. Other sequences were acquired from GenBank (Table 2), and HCoV-OC43 was used as an outgroup

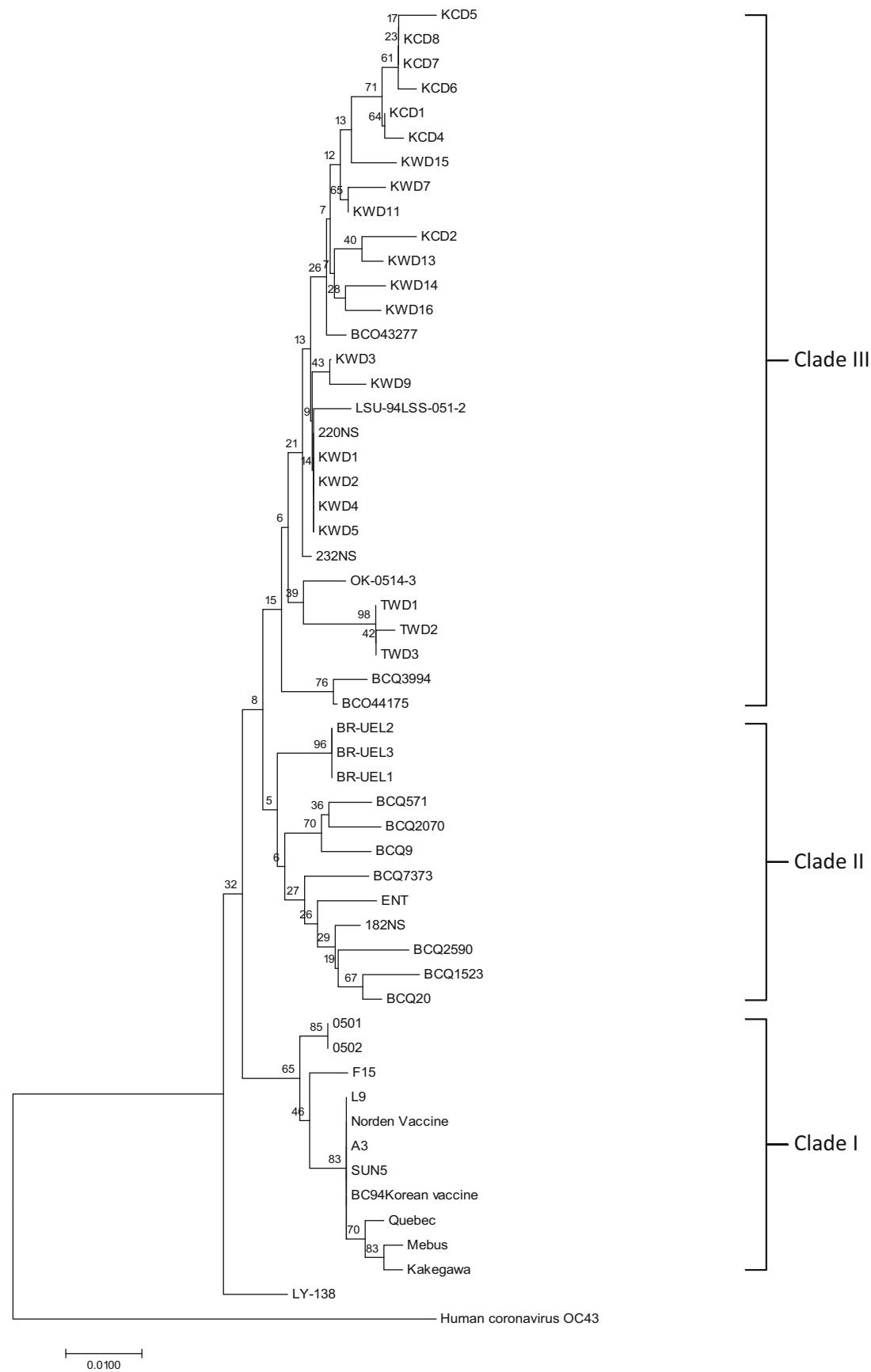

TWD1, TWD2, and TWD3 were closely related to the isolated from three positive samples (99.8 to $100 \%$ similarity), and they also had 98.2 to $98.5 \%$ similarity with OK-0514-3 strain. In Asia, they had 95.9 to $98.5 \%$ similarity with 24 Korean strains and Kakegawa strain. Furthermore, the most distance between those of TWD and other published strains were the Mebus and Kakegawa strain (95.9 to $96.2 \%$ similarity) for the partial S1 gene by nested PCR in Thailand. TWD1, TWD2, TWD3, all of Korean calf diarrhea (KCD) and Korean winter dysentery strains (KWD), some American respiratory strains (OK-0514-3, LSU-94LSS-051-2, 232NS, 220NS), and
Canadian respiratory strains (BCO43277, BCQ3994, BCO44175) were clustered on clade 3. All of the Brazilian strains (BR-UEL1, BR-UEL2, BR-UEL3), Canadian enteric strains (BCQ571, BCQ2070, BCQ9, BCQ1523, BCQ20), Canadian winter dysentery strain (BCQ7373, BCQ2590), American enteric strains (ENT), and American respiratory strains (182NS) were clustered on clade 2 . The Korean vaccine strains(0501, 0502, A3, SUN5, BC94 Korean vaccine), F15, L9, Norden vaccine, Quebec, Mebus, and Kakegawa strain were clustered on clade 1, but LY-138 (American enteric strain) was clustered on a separate branch (Fig. 2). 


\section{Discussion}

In this study, we aimed to identify bovine coronavirus in Thailand and compare our partial S1 sequences with some field and vaccine strains around the world in GenBank. During clinical inspection, the amplicons from nested PCR amplification and percent identity from the BLASTn software of three positive field samples were revealed. This is the first detection of bovine coronavirus in Thailand. Inner primers (Brandao et al. 2003) were able to be detected BCoV. However, the band of PCR with outer primers could not be detected in agarose gel because all of the three positive samples had probably low concentrations of $\mathrm{BCoV}$ or/and PCR inhibitors in feces that may yield false negative. The nested PCR technique will increase more sensitivity and specificity of DNA amplification than conventional PCR technique (Rustempasic et al. 2016).

The phylogenetic tree for the hypervariable region of the $\mathrm{S} 1$ subunit spike glycoprotein $\mathrm{BCoV}$ gene showed that the evolutionally distant, TWD1, TWD2, and TWD3 in our study were the isolated group together and most similar to OK0514-3 strain that is a respiratory strain (RBCoV) (Fig. 2). Respiratory strain was closely related with enteric strain (EBCoV); Hasoksuz et al. (2002) said the BCoV strains may be diverging from an enteric tropism to a dual (respiratory and enteric) tropism over time via intermediates. Moreover, Cho et al. (2000) have observed that gnotobiotic and colostrumdeprived calves inoculated with respiratory isolates from $\mathrm{BCoV}$ in that all strains were pneumoenteric and were shed both nasally and rectally and induced diarrhea. It may explain that there were no variations between the respiratory and the enteric isolates (Hasoksuz et al. 2002). The amino acid substitutions of TWD1, TWD2, and TWD3 from OK-0514-3 strain have been related immunological escape mutation through changes in protein secondary structure (Hasoksuz et al. 2002; Yoo and Deregt 2001). All virulent TWD tended to be distant from the prototype strains, because the allelic variation resulted in genetic mutation over time. Similarly, Kanno et al. (2007) and Fulton et al. (2013) concluded that these isolates had distinctive genetic divergent from the prototype BCoV strains such as Mebus, Quebec, F15, and LY138 strains.

In conclusion, this is the first report of molecular and phylogenetic diagnosis of bovine coronavirus in the dairy cattle from Thailand. It is important, as the data provides that there has been $\mathrm{BCoV}$ infection in Central Region, Thailand. Moreover, phylogenetic tree revealed closely relation between the three isolates, enteric, and respiratory $\mathrm{BCoV}$ strains. Further study will develop ELISA assay for serologic detection of winter dysentery disease.

Acknowledgments This research was financially supported by Kasetsart University 72 Year Anniversary Graduate Scholarship, The
Graduate School, Kasetsart University, Bangkok, Thailand. The authors are thankful to the biotechnology laboratory of the Department of Pathology, Faculty of Veterinary Medicine, Kasetsart University, Kampangsaen Campus, Thailand, for the facilities provided.

\section{Compliance with ethical standards}

Conflict of interest The authors declare that they have no conflict of interest.

\section{References}

Aiumlamai, S, Alenius, S. and Nithichai, K., 1992. Prevalence of antibodies to various bovineviruses in bulk tank milk samples from dairy herds in Muaklek area. Thai Journal of VeterinaryMedicine, 22(2), 112-119.

Blowey, R.W. and Weaver, A.D., 2011. Winter dysentery. Color atlas of disease and disorders of cattle Third edition, 58.

Bosch, B.J., Zee, R.V., Haan, C.A.M., and Rottier, P.J.M., 2003. The coronavirus Spike Protein is a Class I Virus Fusion Protein: Structural and Functional Characterization of the Fusion Core Complex, Journal of Virology, 77, 8801-8811.

Brandao, P.E., Gregori, F., Monteleone, G.S., Soares, R.M., Rosales, C.A., Jerez, R. J.A., 2003.Nested PCR assay for detection of Bovine coronavirus S1 gene. Arquivos do Instituto Biológico,São Paulo, 70(1), 1-3.

Cho, K.O., Halbur, P.G., Bruna, J.D., Sorden, S.D., Yoon,K.J., Janke, B.H., Chang, K.O., Saif, L.J., 2000. Detection and isolation of coronavirus from feces of three herds of feedlot cattle during outbreaks of winter dysentery-like disease. Journal of theAmericanVeterinaryMedicalAssociation, 217, 1191-1194.

Chouljenko, V.N., Lin, X.Q., Storz, J., Kousoulas, K.G. and Gorbalenya, A.E., 2001. Comparison of genomic and predicted amino acid sequences of respiratory and enteric bovine coronaviruses isolated from the same animal with fatal shipping pneumonia. Journal of General Virology, 82,2927-2933.

Fulton, R.W., Ridpath, J.F., and Burge, L.J., 2013. Bovine coronaviruses from the respiratory tract: antigenic and genetic diversity. Vaccine, $31,886-892$.

Graham, R.L., Donaldson, E.F., and Baric, R.S., 2013. A decade after SARS: strategies for controlling emerging coronaviruses. Nature Reviews Microbiology, 11(12), 836-848.

Hasoksuz, M., Sreevatsan, S., Cho, K.O., Hoet, A.E. and Saif, L.J., 2002. Molecular analysis of the S1 subunit of the spike glycoprotein of respiratory and enteric bovine coronavirus isolates.Virus Research, 84, 101-109.

Kanno, T., Hatama, S., Ishihara, R., and Uchida, I., 2007. Molecular analysis of the $\mathrm{S}$ glycoprotein gene of bovine coronaviruses isolated in Japan from 1999 to 2006.Journal ofGeneral Virology, 88, 12181224 .

Masters, P.S.2006. The molecular biology of coronaviruses. Advances in Virus Research, 66, 193-292.

Mebus, C.A., White, R.G., Stair, E.L., Rhodes, M.B. and Twiehaus, M.J., 1972. Neonatal calf diarrhea: results of a field trial using a reo-like virus vaccine. Veterinary Medicine and Small Animal Clinician, 67, 173-178.

Mebus, C.A., Stair, E.L., Rhodes, M.B. and Twiehaus, M.J., 1973. Neonatal calf diarrhea: propagation, attenuation and characteristics of a coronavirus-like agent. American Journal of Veterinary Research, 34, 145-150.

Radostits, O.M., Gay, C.C., Hinchcliff, K.W. and Constable, P.D., 2007. Veterinary medicine-A textbook of the diseases of cattle, horses, 
sheep, pigs, and goats, 10thedition. W. B. Saunders Company Ltd.London, 1286.

Rustempasic, S.M., Mathew, D.E., Alice, L.W. and MichaelD.B., 2016.Sensitive and specific nested PCR assay for detection of rotavirus A in samples with a low viral load. Journal of Virological Methods, 236, 41-46.

Saif, L.J., 2004. Bovine coronavirus infection. Infectious Diseases of Livestock, 2, 795-802.
Weiss, S.R., and Martin, S.N., 2005. Coronavirus pathogenesis and the emerging pathogen severe acute respiratory syndrome coronavirus. Microbiology and Molecular Biology Reviews,69(4), 635-664.

Yoo, D., and DeregtD., 2001. A single amino acid change within antigenic domain II of the Spike protein of Bovine coronavirus confers resistance to virus neutralization. Clinical andDiagnostic Laboratory Immunology, 8(2), 297-302. 